\title{
Gravitational instability of a nonrotating galaxy
}

\author{
Alexander W. Chao \\ SLAC National Accelerator Laboratory, Stanford University, Stanford, California 94309, USA
}

(Received 9 June 2009; published 7 October 2009)

\begin{abstract}
Gravitational instability of the distribution of stars in a galaxy is a well-known phenomenon in astrophysics. This report is an attempt to analyze this phenomenon by applying standard tools developed in accelerator physics. It is found that a nonrotating galaxy would become unstable if its size exceeds a certain limit that depends on its mass density and its temperature.
\end{abstract}

DOI: 10.1103/PhysRevSTAB.12.104201

PACS numbers: 29.20.D-, 95.30.Sf

\section{INTRODUCTION}

There are some notable examples in the past when developments in astrophysics are later found to be profoundly connected to important topics in accelerator physics. It turns out that two of the major topics in the 20th/ 21 st-century accelerator physics-nonlinear dynamics and collective effects-each has its origin traced back to the 19th-century astrophysics.

On the topic of nonlinear dynamics, Henri Poincaré (1854-1912) was believed to be the first who noted the behavior of nonlinear dynamical chaos. In 1887, he entered a contest sponsored by the king of Sweden and Norway, and the problem was to prove that the solar system, as a three-body system, was dynamically stable. He did not succeed in proving it, but his work won the prize anyway. Poincare was also the person who introduced the concept of Poincaré section, which accelerator physicists use every day as they try to describe single-particle motion turn after turn in synchrotrons and storage rings. Indeed, what a beam position monitor detects in these circular accelerators is a special case of Poincaré section. Dynamic aperture and chaotic motion are also typically observed as Poincaré sections - this time on a computer printout instead of an oscilloscope screen connected to a beam position monitor-and have become daily language of nonlinear dynamists in accelerator physics.

On the topic of collective effects, one notable preview from astrophysics was the impressive work by James Clerk Maxwell (1831-1879). In 1857, Maxwell also won a prize - the Adams Prize-when he proved analytically that the Saturn rings cannot be stable unless they consist of many small satellites instead of a single solid piece [1]. Today, we call this Maxwell's mechanism "negative mass instability" in accelerator physics. For accelerators, this Saturn ring instability shows up, for example, in the analysis of the space charge instability.

Following these ground-breaking pioneers, one might ask if today, after years of evolution, there might be some studies that the accelerator physicists have developed, and that can be applied to astrophysics in reciprocation. One such attempt is ventured here. We will try to apply modern accelerator techniques to the well-known problem of a gravitational instability of a galaxy.

The question being asked is: Is a galaxy stable under its own gravitational self-forces? We shall approach this question by the following considerations:

\begin{tabular}{ll}
\hline \hline $\begin{array}{l}\text { Accelerator physics } \\
\text { Collective instability }\end{array}$ & $\begin{array}{c}\text { Astrophysics } \\
\text { Gravitational instability }\end{array}$ \\
\hline $\begin{array}{l}\text { Consider a collection } \\
\text { of charged particles } \\
\text { in a beam }\end{array}$ & $\begin{array}{l}\text { Consider a collection } \\
\text { of stars in a galaxy }\end{array}$ \\
$\begin{array}{l}\text { Force between } \\
\text { particles is } \\
\text { electromagnetic } \\
\text { A beam becomes } \\
\text { unstable when its } \\
\text { charge is too high }\end{array}$ & $\begin{array}{l}\text { Force between stars } \\
\text { is gravitation }\end{array}$ \\
\hline \hline
\end{tabular}

What we will therefore do is to replace beam by galaxy, replace particles by stars, and replace electromagnetic wakefields by gravity.

Gravitational instability of the distribution of stars in a galaxy is a well-known phenomenon; its first analysis appeared almost a century ago under the title of Jeans instability [2]. We can also try to analyze this phenomenon using some of the standard tools developed in accelerator physics [3]. By applying this analysis, it is rederived that a nonrotating galaxy would become unstable if its size exceeds a certain limit that depends on its mass density and its temperature.

Consider a distribution of stars in a galaxy described by a mass-density distribution $\rho(\vec{x}, \vec{v}, t)$ in the phase space $(\vec{x}, \vec{v})$ at time $t$. We wish to analyze the stability of this distribution of stars under the influence of their collective gravitational force. To simplify the problem, we will use a flat Euclidean space-time and will consider Newtonian, nonrelativistic dynamics only. In other words, we ignore both the special theory and the general theory of relativity. The analysis thus does not assume a specific cosmological model other than Newtonian gravity-for example, our calculation does not take into consideration that the uni- 
verse is expanding. If this approach turns out to be fruitful, a large arsenal of analysis tools can be transported from accelerator physics to this and other problems in astrophysics.

The instability we are interested in is self-generated, i.e., it occurs spontaneously. In particular, it does not require an initial "seed" fluctuation at the birth of the galaxy. The instability growth pattern as well as its rate of growth are intrinsic properties of the system.

\section{DISPERSION RELATION}

Consider a particular star in the galaxy. The equations of motion of this star are

$$
\dot{\vec{x}}=\vec{v} \quad \dot{\vec{v}}=G \int d \vec{v}^{\prime} \int d \vec{x}^{\prime} \rho\left(\vec{x}^{\prime}, \vec{v}^{\prime}, t\right) \frac{\vec{x}^{\prime}-\vec{x}}{\left|\vec{x}^{\prime}-\vec{x}\right|^{3}},
$$

where $G$ is the gravitational constant. Note that these equations do not depend on the mass of the star under consideration. Whether we are considering a star or a dust particle in the galaxy does not matter.

Following standard treatment in accelerator physics, evolution of $\rho$ is described by the Vlasov equation [4],

$$
\begin{aligned}
& \frac{\partial \rho}{\partial t}+\frac{\partial \rho}{\partial \vec{x}} \cdot \dot{\vec{x}}+\frac{\partial \rho}{\partial \vec{v}} \cdot \dot{\vec{v}} \\
& =\frac{\partial \rho}{\partial t}+\frac{\partial \rho}{\partial \vec{x}} \cdot \vec{v}+\frac{\partial \rho}{\partial \vec{v}} \cdot G \int d \vec{v}^{\prime} \int d \vec{x}^{\prime} \rho\left(\vec{x}^{\prime}, \vec{v}^{\prime}, t\right) \frac{\vec{x}^{\prime}-\vec{x}}{\left|\vec{x}^{\prime}-\vec{x}\right|^{3}} \\
& =0 .
\end{aligned}
$$

Equation (2) is a nonlinear, partial differential, integral equation for $\rho$. It is nonlinear because the third term is quadratic in $\rho$. Our job is to solve for $\rho$.

To examine the stability of the system, let the galaxy distribution be given by an unperturbed distribution $\rho_{0}$ plus some small perturbation. Let the unperturbed distribution $\rho_{0}$ depend only on $\vec{v}$,

$$
\rho_{0}=\rho_{0}(\vec{v}) .
$$

In particular, $\rho_{0}$ does not depend on $\vec{x}$ or $t$. This unperturbed distribution is uniform in $\vec{x}$, i.e., it is uniform in the infinite 3D space. The function $\rho_{0}(\vec{v})$ is so far unrestricted. This model is the 3D analogue of the 1D "coasting beam" or "unbunched beam" in accelerator physics.

Our approach to analyze the problem is based on the following scheme. We will allow the small perturbation around $\rho_{0}$ to depend on $t$ and $\vec{x}$, although the unperturbed distribution $\rho_{0}$ has been assumed to be independent of $t$ and $\vec{x}$. We consider a situation when the galaxy distribution contains a slight but otherwise arbitrary deviation from $\rho_{0}$ at some initial moment of time. There are of course an infinite number of possible initial deviations, but for any one initial deviation, we want to know how it evolves with time. For the galaxy to be stable, the deviation must not grow in time for all possible initial deviations. If the deviation grows in time for any initial deviation, even if one finds only one such example, then the galaxy is considered to be unstable.

We refer to the deviation from $\rho_{0}$ as a perturbation to galaxy distribution. To analyze the problem, we Fourier decompose the perturbation and write

$$
\rho(\vec{x}, \vec{v}, t)=\rho_{0}(\vec{v})+\Delta \rho(\vec{v}) e^{-i \omega t+i \vec{k} \cdot \vec{x}},
$$

where $\vec{k}$ is the wave number vector and $\omega$ is the angular oscillation frequency of the perturbation; $\vec{k}$ characterizes the spatial behavior of the perturbation while $\omega$ describes its time behavior. We anticipate that for a given $\vec{k}$, there will be a specific oscillation frequency $\omega$ [5].

An examination of the proposed analysis scheme then leads to the conclusion that we should consider $\vec{k}$ as a real quantity because it describes the initial spatial distribution of $\Delta \rho$, while $\omega$ should in general be a complex quantity because it must allow us to describe a perturbation growing exponentially in time. With the time dependence of the perturbation given by $\sim e^{-i \omega t}$ as prescribed in Eq. (4), we see that the imaginary part of $\omega$ is the instability growth rate [growth rate if $\operatorname{Im}(\omega)>0$, damping rate if $\operatorname{Im}(\omega)<$ $0]$. Our job is then to find the complex $\omega(\vec{k})$ as a function of an arbitrary real $\vec{k}$. If we find for any $\vec{k}$ that its corresponding $\operatorname{Im}[\omega(\vec{k})]$ is positive, the galaxy is unstable.

Substituting Eq. (4) into Eq. (2) and keeping only first order in $\Delta \rho$ (linearize with respect to $\Delta \rho$ ) yields

$$
-i(\omega-\vec{v} \cdot \vec{k}) \Delta \rho(\vec{v})+G\left(\int d \vec{v}^{\prime} \Delta \rho\left(\vec{v}^{\prime}\right)\right) \frac{\partial \rho_{0}(\vec{v})}{\partial \vec{v}} \cdot \vec{q}(\vec{k})=0,
$$

where the field generated by $\rho_{0}$ has been neglected assuming it vanishes due to the perfect uniformity in its spatial distribution, and

$$
\vec{q}(\vec{k}) \equiv \int d \vec{x}^{\prime} \frac{e^{i \vec{k} \cdot\left(\vec{x}^{\prime}-\vec{x}\right)}\left(\vec{x}^{\prime}-\vec{x}\right)}{\left|\vec{x}^{\prime}-\vec{x}\right|^{3}}=\int d \vec{y} \frac{e^{i \vec{k} \cdot \vec{y}} \vec{y}}{|\vec{y}|^{3}}
$$

is a well-defined quantity depending only on $\vec{k}$; it is the Fourier transform of the Newton kernel $\vec{x} /|\vec{x}|^{3}$, and might be called the "graviton propagator" following a terminology in quantum field theory. In fact, aside from the singularity at the origin $\vec{k}=\overrightarrow{0}$, it can be shown that

$$
\vec{q}(\vec{k})=\frac{4 \pi i}{|\vec{k}|^{2}} \vec{k}
$$

In accelerator physics, the Newton kernel $\vec{x} /|\vec{x}|^{3}$ stands for the "wake function" while its Fourier transform $\vec{q}$ stands for the "impedance" [3].

Equation (5) can be rewritten as

$$
\Delta \rho(\vec{v})=-i G\left(\int d \vec{v}^{\prime} \Delta \rho\left(\vec{v}^{\prime}\right)\right) \frac{\frac{\partial \rho_{0}(\vec{v})}{\partial \vec{v}} \cdot \vec{q}(\vec{k})}{\omega-\vec{v} \cdot \vec{k}} .
$$

Integrating both sides over $\vec{v}$ and canceling out the com- 
mon factor of $\int d \vec{v}^{\prime} \Delta \rho\left(\vec{v}^{\prime}\right)$ then gives a dispersion relation that must be satisfied by $\omega$ and $\vec{k}$,

$$
1=-i G \int d \vec{v} \frac{\frac{\partial \rho_{0}(\vec{v})}{\partial \vec{v}} \cdot \vec{q}(\vec{k})}{\omega-\vec{v} \cdot \vec{k}} .
$$

Given $\rho_{0}(\vec{v})$, we solve this dispersion relation for $\omega$ as a function of $\vec{k}$. This solution is then used to find the most unstable pattern of perturbation and its corresponding growth rate, as will be described next.

\section{UNIFORM ISOTROPIC GALAXY}

We next consider an unperturbed distribution that depends only on the magnitude of $\vec{v}$, i.e., let

$$
\rho_{0}=\rho_{0}\left(|\vec{v}|^{2}\right)
$$

which gives

$$
\frac{\partial \rho_{0}}{\partial \vec{v}}=2 \vec{v} \rho_{0}^{\prime}\left(|\vec{v}|^{2}\right)
$$

This is the case of a uniform isotropic (uniform in $\vec{x}$, isotropic in $\vec{v}$ ) galaxy. The normalization condition is

$$
\begin{aligned}
& \int_{0}^{\infty} 4 \pi v^{2} d v \rho_{0}\left(v^{2}\right) \\
& =\rho_{m}=\text { mass density of stars per unit volume. }
\end{aligned}
$$

Substituting Eqs. (7) and (11) into Eq. (9) then gives

$$
1=\frac{8 \pi G}{|\vec{k}|^{2}} \int d \vec{v} \rho_{0}^{\prime}\left(|\vec{v}|^{2}\right) \frac{\vec{v} \cdot \vec{k}}{\omega-\vec{v} \cdot \vec{k}} .
$$

Letting $\vec{k}=(0,0, k)$, and choosing coordinates so that $\vec{v}=v(\sin \theta \cos \phi, \sin \theta \sin \phi, \cos \theta)$, Eq. (13) becomes, with a change of variable $u=\cos \theta$,

$$
1=\frac{16 \pi^{2} G}{k} \int_{0}^{\infty} v^{3} d v \rho_{0}^{\prime}\left(v^{2}\right) \int_{-1}^{1} d u \frac{u}{\omega-k v u} .
$$

One must refrain from performing the integration over $u$ at this time because that integral involves a singularity. Proper treatment of the singularity follows the standard technique used in accelerator physics (and plasma physics) on Landau damping [6].

Landau damping is a general phenomenon that occurs in several branches of physics. It has a specific interpretation in accelerator physics, but its physical picture as well as its mathematical manipulations are the same as in other fields. To summarize, the treatment amounts to adding an infinitesimal positive imaginary part to $\omega$, i.e. $\omega \rightarrow \omega+i \epsilon$. The integral involving the singularity,

$$
I(\omega, k v) \equiv \int_{-1}^{1} d u \frac{u}{\omega-k v u} \rightarrow \int_{-1}^{1} d u \frac{u}{\omega+i \epsilon-k v u},
$$

becomes an integration along the contour $\mathcal{C}_{1}$ as in Fig. 1(a) where $\mathcal{C}_{1}$ is drawn in a complex plane of $u$ and the integration is along the real axis of $u$. The singularity, a (a)

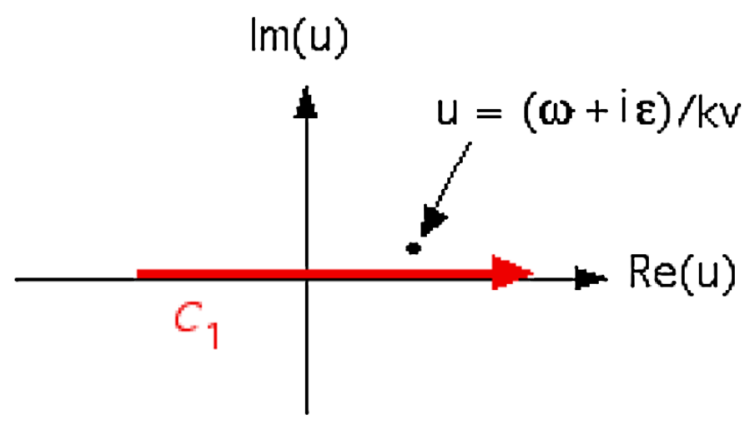

(b)

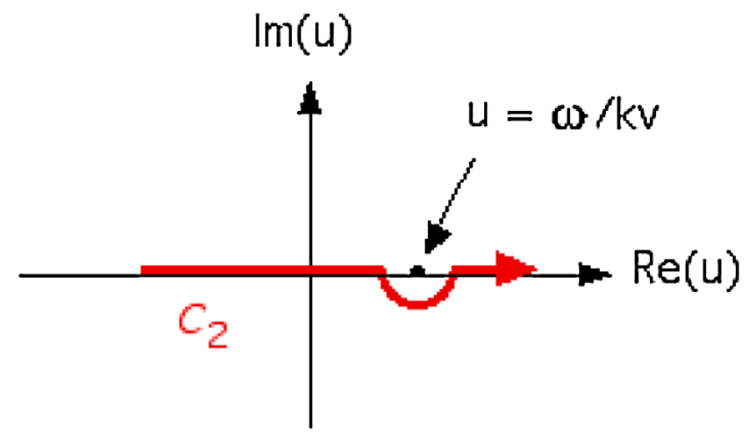

FIG. 1. (Color) Integration contour dictated by Landau damping analysis. The quantity $\epsilon$ in (a) and the radius of the semicircle in (b) are considered to be infinitesimal.

pole, is displaced above the real axis for the case when $k v>0$ as indicated.

Using complex variable analysis, we know we can deform the integration contour from $\mathcal{C}_{1}$ to $\mathcal{C}_{2}$, as shown in Fig. 1(b). Around the singularity pole, the contour $\mathcal{C}_{2}$ traces out a perfect, left-right symmetric, infinitesimally small, semicircle.

We now can calculate the integral $I(\omega, k v)$. It contains two parts: part 1 from integration along the real axis of $\mathcal{C}_{2}$ and part 2 from the contribution of the semicircle. The result is

$$
\begin{aligned}
I(\omega, k v)= & \text { P.V. } \int_{-1}^{1} d u \frac{u}{\omega-k v u}-\frac{i \pi \omega}{k^{2} v^{2}} H\left(1-\left|\frac{\omega}{k v}\right|\right) \\
= & -\frac{2}{k v}-\frac{\omega}{k^{2} v^{2}} \ln \left|\frac{\omega-k v}{\omega+k v}\right| \\
& -\frac{i \pi \omega}{k^{2} v^{2}} H\left(1-\left|\frac{\omega}{k v}\right|\right),
\end{aligned}
$$

where P.V. means taking the principal value of the integral, and $H(x)=1$ for $x>0$ and 0 for $x<0$ is the step function. The P.V. term comes from part 1 of the integral while the step function term comes from part 2. By taking P.V., the singularity in the integral over $u$ is avoided in a wellbehaved manner [7]. Equation (15) gives explicit calculated result of this P.V. term.

As to the step function term, we note that a full circle around the pole gives a contribution of $2 \pi i$ times the residue of the pole; so this semicircle contributes just 
half as much. Note that this contribution is imaginary, and as a result, the quantity $\int_{-1}^{1} d u \frac{u}{\omega-k v u}$ is attributed an imaginary part. Furthermore, this extra imaginary term has a definitive sign (proportional to $-i \omega$ ). This particular sign is such that this mechanism is Landau damping and not Landau antidamping.

To be specific, we next take a uniform distribution of $\rho_{0}$ (uniform in $\vec{x}$, isotropic in $\vec{v}$, and $\rho$ is constant up to $v_{0}$ ),

$$
\rho_{0}\left(v^{2}\right)= \begin{cases}\frac{3 \rho_{m}}{4 \pi v_{0}^{3}} & \text { if } v^{2}<v_{0}^{2} \\ 0 & \text { otherwise }\end{cases}
$$

This distribution is very close (but not identical) to what is called the "water-bag model" in accelerator physics. The quantity $v_{0}^{2}$ is related to the "temperature" of the galaxyas will be discussed more later. Substituting Eqs. (16) and (15) into Eq. (14) gives the dispersion relation

$$
\lambda=\frac{1}{2+x \ln \left|\frac{x-1}{x+1}\right|+i \pi x H(1-|x|)}
$$

where

$$
\lambda=\frac{6 \pi G \rho_{m}}{k^{2} v_{0}^{2}} \quad \text { and } \quad x=\frac{\omega}{k v_{0}} .
$$

In accelerator physics, $\lambda$ is replaced by the impedance. One simplification for the gravitational instability is that $\lambda$ is a real quantity, while the impedance is complex in general.

\section{STABILITY CONDITION}

We next need to compute the instability growth rate, which is given by the imaginary part of $\omega$, as a function of $k$. The star distribution $\rho_{0}(\vec{v})$ would be unstable if, for any $\vec{k}$, its corresponding $\omega$ is complex with a positive imaginary part. We need to compute $x$ as a function of $\lambda$ using Eq. (17) in order to obtain $\omega$ as a function of $k$. Unfortunately Eq. (17) gives $\lambda$ as a function of $x$, and its inversion to give $x$ as a function of $\lambda$ is difficult. To deal with this difficulty, we apply another technique of accelerator physics as follows.

In general $x$ is complex, but at the edge of instability when the system is barely unstable, $x$ is real. The edge of stability can therefore be seen by plotting the right-hand side of Eq. (17) as $x$ is scanned along the real axis from $-\infty$ to $\infty$. Figure 2 shows the real and imaginary parts of the right-hand side of Eq. (17) in such a scan. The horizontal and vertical axes are the real and imaginary parts of the right-hand side of Eq. (17), respectively. As $x$ is scanned from $-\infty$ to $\infty$, the right-hand side of Eq. (17) traces out a cherry-shaped diagram-including the "stem" of the cherry running from $-\infty$ to 0 along the real axis. This cherry curve defines the boundary between stable and unstable regions. If $\lambda$, the left-hand side of Eq. (17), lies inside this cherry diagram (including the stem), the galaxy distribution is stable. If it lies outside, the galaxy is unstable. Since $\lambda$ is necessarily real and positive, the stability

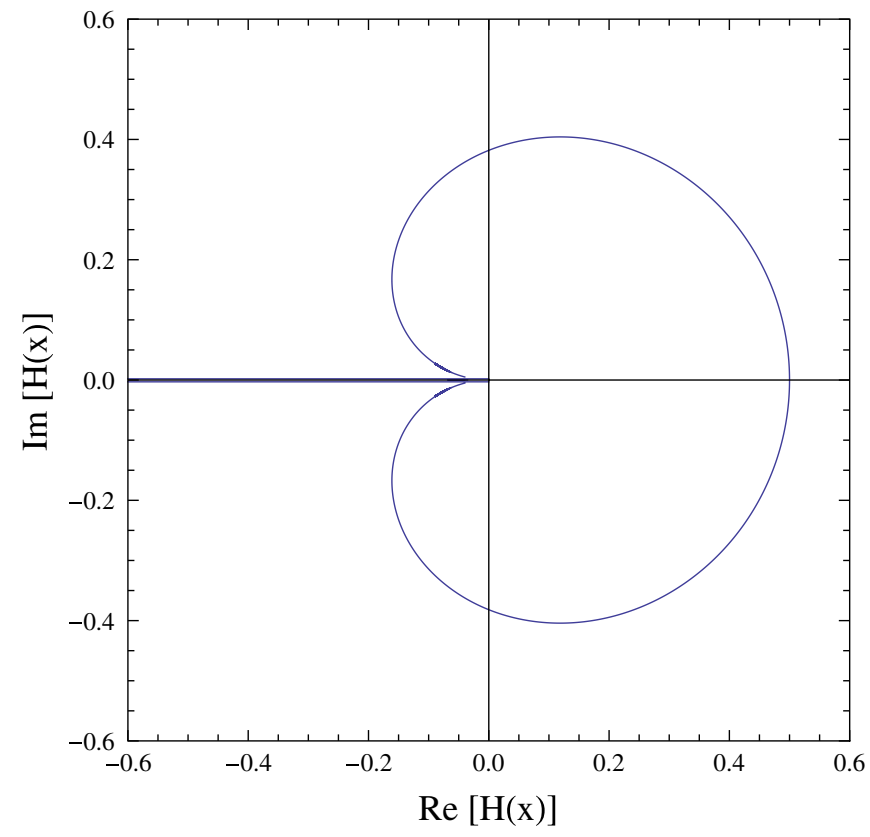

FIG. 2. (Color) Stability diagram for the galaxy distribution, where $H(x)$ is the right-hand side of Eq. (17).

condition therefore reads, very simply,

$$
\lambda<\frac{1}{2}
$$

One may ask what the corresponding result of Eq. (19) is in accelerator physics. The answer is that it gives rise to a condition that $\left|Z_{0}^{\|} / n\right|$ should be less than some threshold value, where $\left|Z_{0}^{\|} / n\right|$ is related to the impedance. This stability condition in accelerator physics is given the name "Keil-Schnell criterion" [8].

Equation (19) indicates that a hot galaxy (high temperature, i.e., large $v_{0}$ ) is more stable than a cold one. This is expected due to the Landau damping mechanism. It also indicates that the star distribution is unstable for longwavelength perturbations (small $k$ ). The threshold wavelength is given by

$$
x_{\mathrm{th}}=\frac{2 \pi}{k_{\mathrm{th}}}
$$

where

$$
k_{\mathrm{th}}=\frac{\sqrt{12 \pi G \rho_{m}}}{v_{0}} .
$$

Perturbations with wavelengths longer than $x_{\text {th }}$ are unstable. One might expect that the galaxy will have a dimension of the order of $x_{\text {th }}$ because, if the galaxy had a larger dimension, it would have broken up due to the instability until it is reduced to the stable size. 


\section{PRESSURE AND TEMPERATURE OF THE GALAXY}

It might be instructive to relate to an internal "pressure" of the galaxy distribution by pretending the galaxy is a free gas bounded by some container, with the gas particles bouncing off the container walls with perfect reflection. It is easy to show that the pressure exerted onto the container wall is given by

$$
P=\frac{2}{3} \rho_{m}\left\langle v^{2}\right\rangle=\frac{8 \pi}{3} \int_{0}^{\infty} v^{4} d v \rho_{0}\left(v^{2}\right) .
$$

For the uniform distribution (16), it gives an internal pressure of our galaxy,

$$
P=\frac{2}{5} \rho_{m} v_{0}^{2}
$$

We can also rewrite the galaxy's stability condition (19) in terms of its pressure. Stability then requires

$$
\text { (galaxy dimension) } \times \rho_{m}<\sqrt{\frac{5 \pi}{6} \frac{P}{G}} \text {. }
$$

Pushing one step further, one might be tempted also to relate to a temperature of the galaxy. To do this, however, it is now necessary to define the mass of an individual star. Let all stars have mass $m$, then the galaxy's temperature is

$$
T=\frac{m}{k_{B}} \frac{P}{\rho_{m}},
$$

where $k_{B}$ is the Boltzmann constant.

Since a galaxy's size is proportional to $v_{0}$ [see Eqs. (20) and (21)], it is then proportional to the square root of its temperature for a given $\rho_{m}$. Equivalently, the temperature of a stable galaxy is proportional to the square of its size,

$$
T \propto(\text { galaxy size })^{2} .
$$

\section{INSTABILITY GROWTH RATE}

When $\lambda>1 / 2, \omega$ will be complex. The instability growth rate is determined by the imaginary part of $\omega$,

$$
\tau^{-1}=\operatorname{Im}(\omega) .
$$

We need to modify Eq. (17) slightly for complex $\omega$. In the unstable region, let

$$
\frac{\omega}{k v_{0}}=x+i y, \quad(y>0)
$$

Equation (17) reads

$$
\begin{aligned}
\frac{1}{\lambda}= & +\left(\frac{x+i y}{2}\right) \ln \left[\frac{(x-1)^{2}+y^{2}}{(x+1)^{2}+y^{2}}\right]+(i x-y) \\
& \times\left[\tan ^{-1}\left(\frac{x+1}{y}\right)-\tan ^{-1}\left(\frac{x-1}{y}\right)\right] .
\end{aligned}
$$

When $y \rightarrow 0^{+}$, we obtain Eq. (17) as it should.
Note that the right-hand side of Eq. (25) has the property that its real part is an even function and its imaginary part is an odd function of $y$. However, one should keep in mind that our analysis applies only when $y>0$. This is because we have demanded $\omega$ to have a positive imaginary part when we made the Landau criterion (15). In contrast, the variable $x$ runs from $-\infty$ to $+\infty$.

We will need to solve Eq. (25) for $x$ and $y$ for given $\lambda>$ $\frac{1}{2}$. It turns out that in this range there is always one solution with purely imaginary $\omega$, i.e. $x=0$, and therefore

$$
\lambda=\frac{1}{2-2 y \tan ^{-1}\left(\frac{1}{y}\right)}
$$

or, written out explicitly,

$$
\frac{6 \pi G \rho_{m}}{k^{2} v_{0}^{2}}=\frac{1}{2-\frac{2 \tau^{-1}}{k v_{0}} \tan ^{-1}\left(\frac{k v_{0}}{\tau^{-1}}\right)} .
$$

We need to find $\tau^{-1}$ as a function of $k$. To do so, we first scale the variables by

$$
u=\frac{k v_{0}}{\sqrt{6 \pi G \rho_{m}}}, \quad v=\frac{\tau^{-1}}{\sqrt{6 \pi G \rho_{m}}}
$$

and then

$$
\frac{1}{u^{2}}=\frac{1}{2-2\left(\frac{v}{u}\right) \tan ^{-1}\left(\frac{u}{v}\right)} .
$$

Figure 3 shows the result of $v$ versus $u$.

As seen from Fig. 3, the growth rate vanishes $(v=0)$ when $u=\sqrt{2}$, corresponding to $\lambda=1 / 2$, i.e., at the stability boundary. This is of course expected. Figure 3 also shows that instability occurs fastest for small $u$, i.e., small $k$

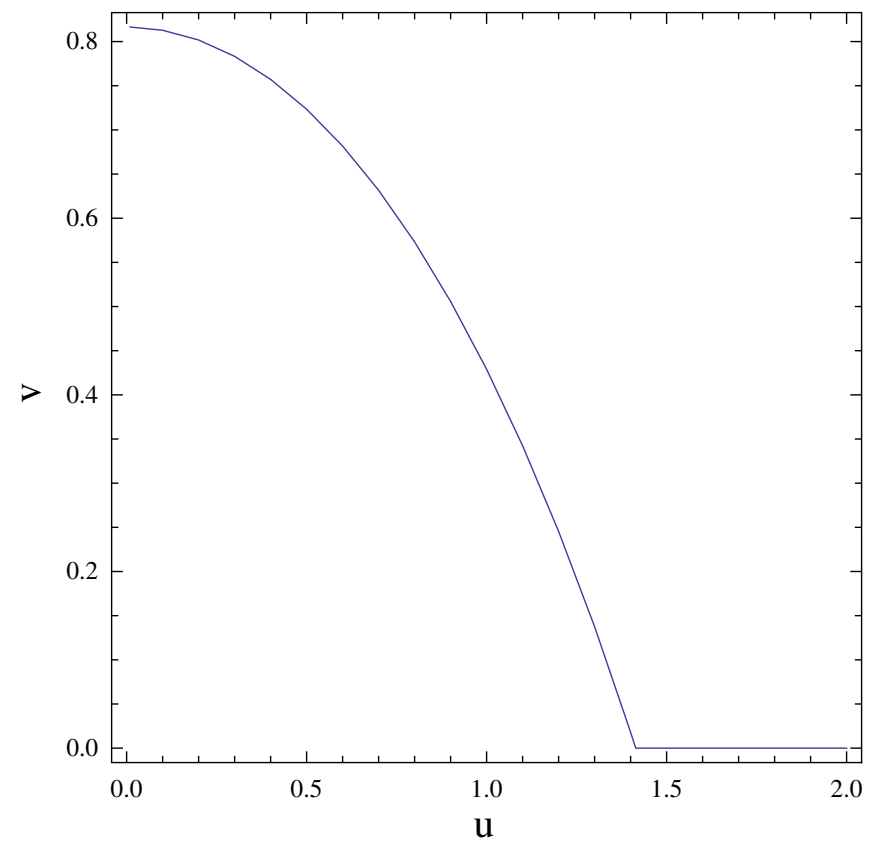

FIG. 3. (Color) $v$ vs $u$ according to Eq. (29). 


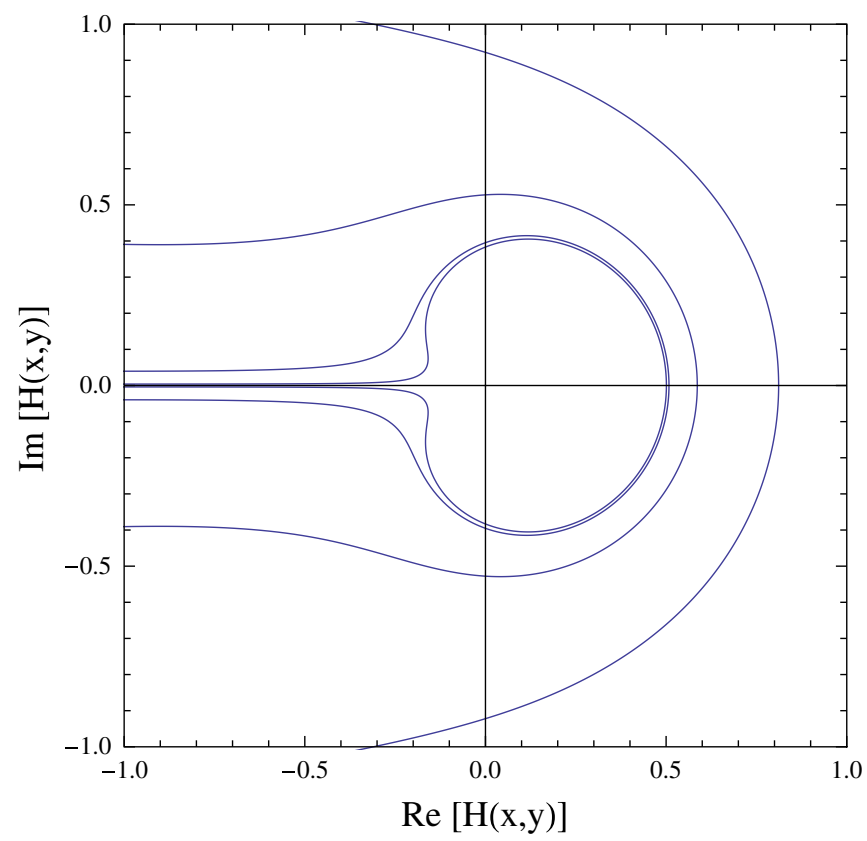

FIG. 4. (Color) Contours of constant growth rates, where $H(x, y)$ is the right-hand side of Eq. (25).

or large wavelength of the perturbation. The growth rate is maximum at $u=0$ with $v=\sqrt{2 / 3}$. This means the maximum growth rate occurs for perturbation of infinite wavelength, and is given by

$$
\left(\tau^{-1}\right)_{\max }=\sqrt{4 \pi G \rho_{m}} .
$$

Note that the growth rate is independent of $v_{0}$, even though that for instability, there is still the condition $\lambda>$ $1 / 2$, which does depend on $v_{0}$ and can be cast into the form [see Eq. (21)]

$$
k<\frac{\sqrt{3}}{v_{0}}\left(\tau^{-1}\right)_{\max }
$$

The result that fastest instability occurs for perturbations of infinitely long wavelength $(k=0)$ depends on our assumption of Newtonian dynamics of action at a distance. Under this assumption, perturbation at one location instantly affects locations infinitely far away. If this assumption is appropriately removed, it is expected that the instability for perturbations with very large wavelengths will be weakened if $v_{0}$ approaches the speed of light $c$.
According to Eq. (31), stable galaxies must have a dimension smaller than a critical value, i.e.

$$
\text { galaxy dimension }<\frac{2 \pi v_{0}}{\sqrt{12 \pi G \rho_{m}}} .
$$

Stability is provided through Landau damping. When the temperature $T \rightarrow 0$, no galaxies can be stable. Equations (30) and (32) are our main results.

Figure 4 shows four traces; each is the locus of the stability contour when $x$ is scanned from $-\infty$ to $\infty$ while $y$ is held fixed. The four traces correspond, starting with the inner most one, to $y=0.001,0.01,0.1$, and 0.3 . When $y=$ 0, the trace reproduces Fig. 2.

\section{OTHER ISOTROPIC DISTRIBUTIONS}

So far we have assumed a somewhat idealized water-bag distribution (16) for the galaxy's temperature. One can improve it slightly by considering other distributions. For a general isotropic distribution, it is a few simple algebraic steps following Eq. (14) to derive the dispersion relation,

$\lambda=\frac{-3 \rho_{m} /\left(8 \pi v_{0}^{3}\right)}{\int_{0}^{\infty} v d v \rho^{\prime}\left(v^{2}\right)\left(\frac{2 v}{v_{0}}+x \ln \left|\frac{x v_{0}-v}{x v_{0}+v}\right|\right)+i \pi x \int_{|x| v_{0}}^{\infty} v d v \rho^{\prime}\left(v^{2}\right)}$,

where $\lambda$ and $x$ are given by Eq. (18).

Four examples are given below:

Case 1

$$
\rho\left(v^{2}\right)=\frac{\rho_{m} v_{0}}{\pi^{2}\left(v^{2}+v_{0}^{2}\right)^{2}} \Rightarrow \lambda=\frac{3 / 2}{\frac{1-x^{2}}{\left(1+x^{2}\right)^{2}}+i \frac{2 x}{\left(1+x^{2}\right)^{2}}}
$$

Case 2

$$
\begin{aligned}
\rho\left(v^{2}\right) & =\frac{3 \rho_{m} v_{0}^{2}}{4 \pi\left(v^{2}+v_{0}^{2}\right)^{5 / 2}} \Rightarrow \lambda \\
& =\frac{3 / 4}{\frac{2-x^{2}}{2\left(1+x^{2}\right)^{2}}-\frac{3 x \sinh ^{-1} x}{2\left(1+x^{2}\right)^{5 / 2}}+i \frac{3 \pi x}{4\left(1+x^{2}\right)^{5 / 2}}}
\end{aligned}
$$

Case 3

$$
\rho\left(v^{2}\right)=\frac{4 \rho_{m} v_{0}^{3}}{\pi^{2}\left(v^{2}+v_{0}^{2}\right)^{3}} \Rightarrow \lambda=\frac{1 / 2}{\frac{3-6 x^{2}-x^{4}}{3\left(1+x^{2}\right)^{3}}+i \frac{8 x}{3\left(1+x^{2}\right)^{3}}}
$$

Case $4 \quad \rho_{0}\left(v^{2}\right)=\frac{\rho_{m}}{4 \pi \sqrt{2 \pi} v_{0}^{3}} e^{-v^{2} / 2 v_{0}^{2}} \Rightarrow \lambda=\frac{3 \sqrt{2 \pi}}{\int_{0}^{\infty} y d y e^{-\left(y^{2} / 2\right)}\left(2 y+x \ln \left|\frac{x-y}{x+y}\right|\right)+i \pi x \int_{|x|}^{\infty} y d y e^{-\left(y^{2} / 2\right)}}$.

Figure 5 shows the stability diagrams for the various cases. For the galaxy to be stable, the parameter $\lambda$ must be less than $\frac{3}{2}, \frac{3}{4}, \frac{1}{2}$, and 3 , for cases $1,2,3$, and 4 , respectively, noting the fact that only the positive real values of $\lambda$ are meaningful. 

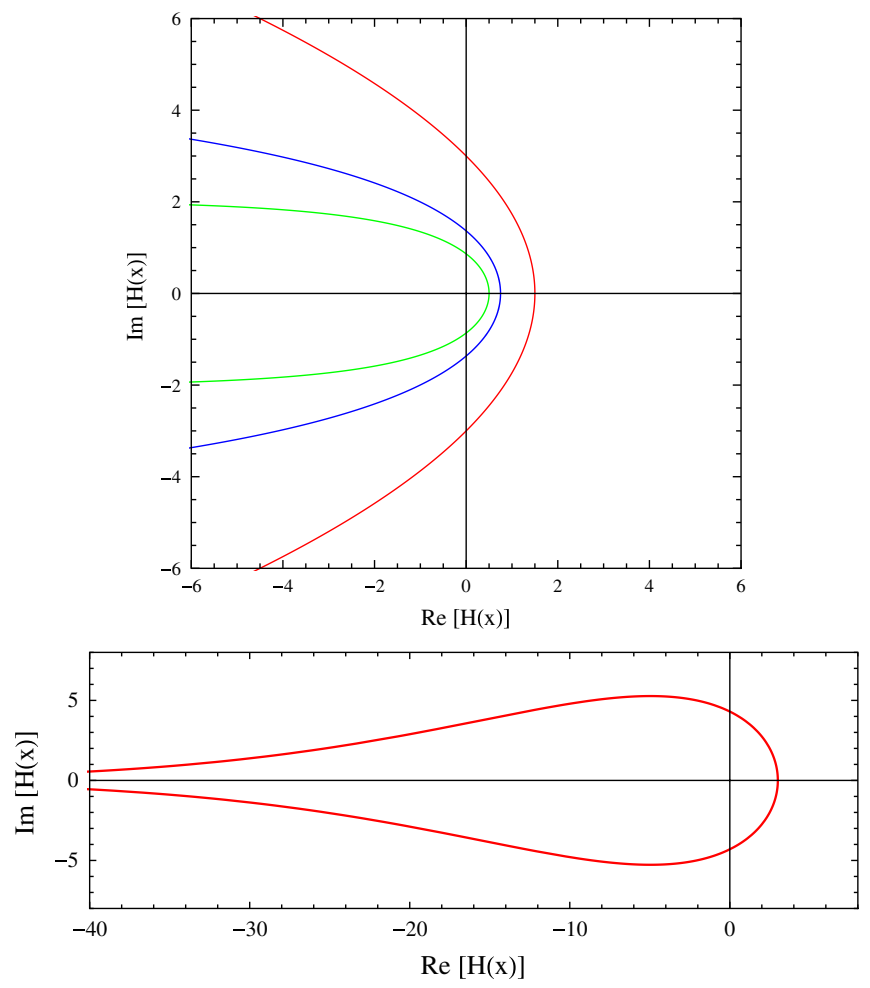

FIG. 5. (Color) (Upper) Stability diagrams for galaxy distributions are plotted in red, blue, and green for Cases 1, 2, and 3, respectively. (Lower) Case 4: Function $H(x)$ represents the righthand sides of the dispersion relations for the four cases.

\section{NUMERICAL ESTIMATES AND DISCUSSIONS}

For a numerical application, we take estimates from the Milky Way [2,9],

$$
\rho_{m}=2 \times 10^{-23} \mathrm{~g} / \mathrm{cm}^{3}, \quad v_{0}=200 \mathrm{~km} / \mathrm{s} .
$$

Using the result of the water-bag model, we obtain a shortest growth time of $7 \times 10^{6}$ years for perturbations with very long wavelengths. For stability, the galaxy dimension must be smaller than 14000 light-years, which seems to be consistent with the size of the Milky Way.

It is conceivable that the same analysis can be applied to the dynamics of galaxies in a galaxy cluster, instead of stars in a galaxy. In that case, $\rho(\vec{x}, \vec{v}, t)$ describes the distribution of galaxies in the galaxy cluster. We might then take

$$
\rho_{m}=10^{-28} \mathrm{~g} / \mathrm{cm}^{3}, \quad v_{0}=1000 \mathrm{~km} / \mathrm{s} .
$$

We obtain a growth time of $3 \times 10^{9}$ years. The galaxy cluster dimension should be smaller than $3 \times 10^{7}$ lightyears. These values do not seem to be unreasonable.

For completeness, we give a few further discussions below [10].

(i) The case studied so far is that of a galaxy initially with infinite size. One direction to extend this study is to consider finite-sized galaxies. In Appendix A, a 1D nonrotating galaxy model has been implemented for this purpose. In this model, each star is an infinite plane and is allowed to move only in the $z$ dimension. The galaxy's $z$ dimension size is finite. An unperturbed distribution with finite temperature to balance out the gravitational pull is found first. The temperature turns out to be sufficient to provide stability to the galaxy by the Landau damping mechanism. A computer code was written to simulate the motion of stars in this galaxy. The result is shown in Fig. 6.

(ii) We have also implemented a $2 \mathrm{D}$ rotating model in Appendix B. In this model, each star is a line mass infinitely long in the $z$ dimension and free to move in the $x$ and $y$ dimensions. This galaxy has a finite size in $x$ and $y$ dimensions. An unperturbed distribution is found when the rotating centrifugal force exactly balances the gravitational pull. This rotation requires stars to have different initial velocities. It turns out that the corresponding velocity spread is sufficient to stabilize the galaxy. A simulation of this galaxy is shown in Fig. 7.

(iii) Application can be extended to two colliding galaxies, drawing analogy to the two stream instabilities in accelerator physics. A small accidental ripple in the density distribution in one galaxy gets imprinted onto the oncoming galaxy; the perturbation on the second galaxy then enhances the initial ripple on the first galaxy by gravitational interaction, leading to instability.

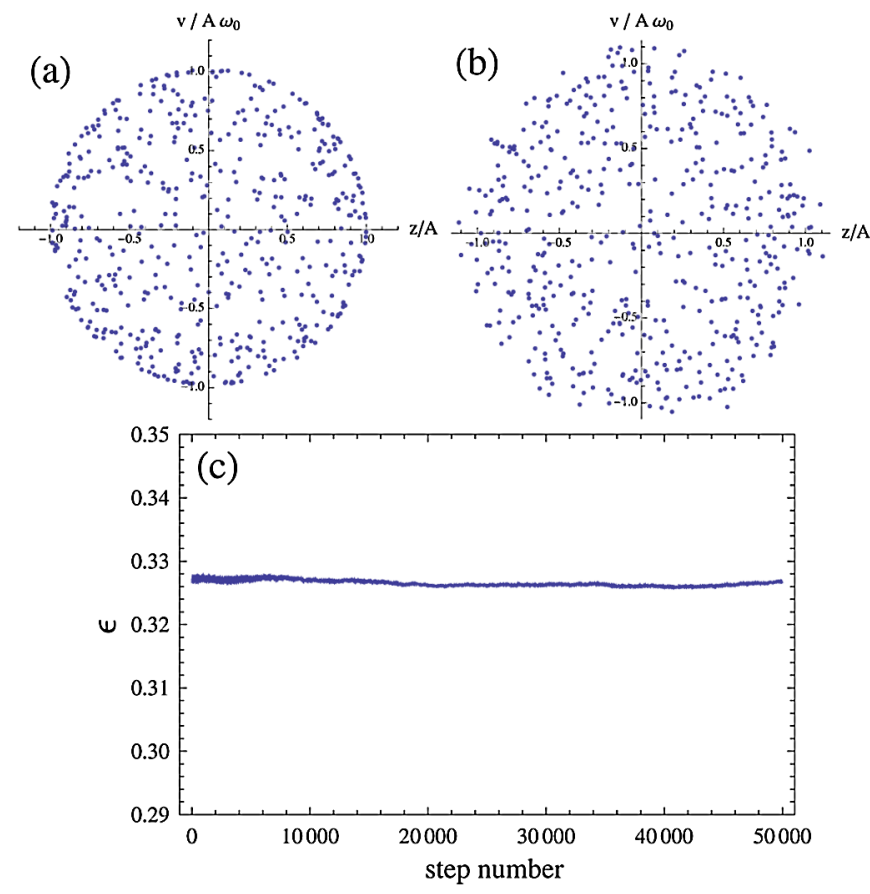

FIG. 6. (Color) Simulation result of a 1D nonrotating galaxy model: (a) Initial phase space distribution of 500 stars; (b) final distribution after $2000 / 2 \pi$ periods; (c) plot of galaxy emittance $\epsilon=\sqrt{\left[\left(v / \omega_{0} A\right)^{2}+(z / A)^{2}\right] / 2}$ as a function of the step number $t / \Delta t$, showing no sign of instability. 


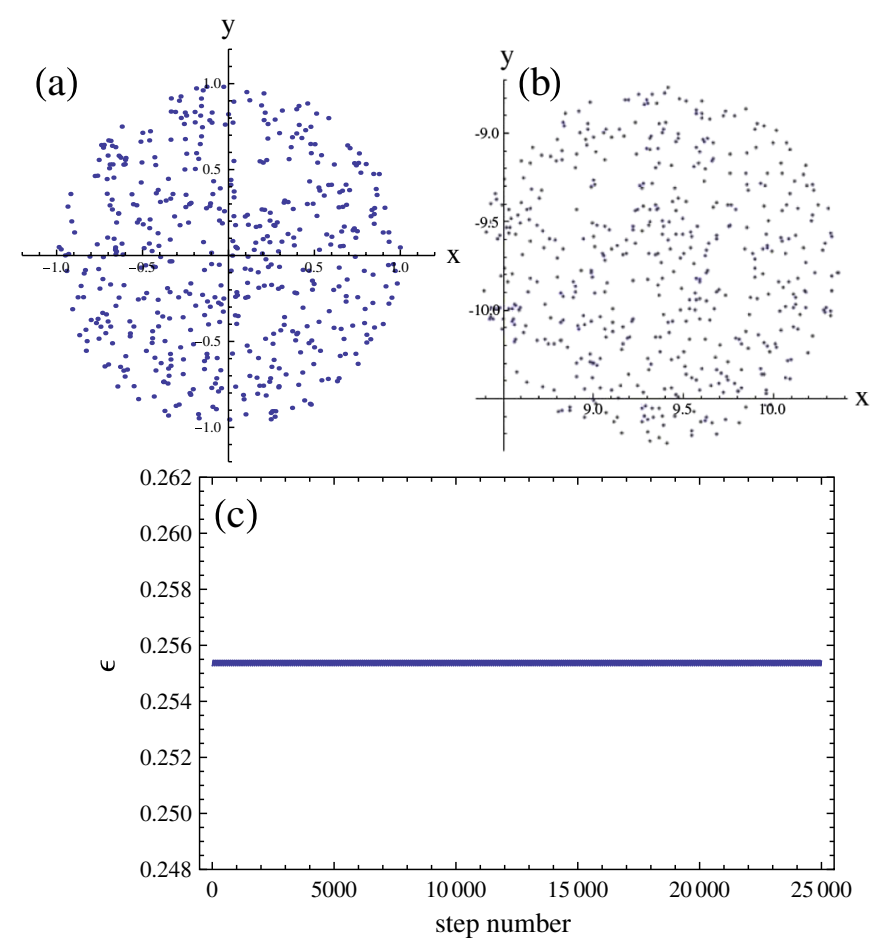

FIG. 7. (Color) Simulation result of the 2D rotating galaxy: (a) initial distribution of 500 stars in the $x-y$ space; (b) final distribution after $1000 / 2 \pi$ periods; (c) plot of galaxy emittance $\epsilon=\sqrt{\left[\left(\dot{x} / \omega_{0} A\right)^{2}\right] / 2+(x / A)^{2}}$ as a function of the step number $t / \Delta t$, showing no sign of instability.

(iv) Still further extensions might take into account the special relativity and general relativity to replace Newtonian gravity. The special theory of relativity will circumvent the action-at-a-distance problem. To include general relativity, the space-time metrics will have to be modified. By extending to this non-Euclidean space-time, one may conceive a study of this gravitational instability mechanism for particles near a large black hole.

\section{ACKNOWLEDGMENTS}

This work was supported by Department of Energy Contract No. DE-AC02-76SF00515.

\section{APPENDIX A: A 1D NONROTATING GALAXY MODEL}

In the text, we have discussed a 3D isotropic galaxy model which initially has an infinite uniform extent. Here we examine the stability of a 1D model which initially has a finite size. In this 1D model, each star is really a thin planar sheet of mass with infinite size in $x$ and $y$ dimensions. Each sheet is free to move only in the $z$ direction. Let the mass distribution of these stars be given by $\rho(z, v, t)$ in the $(z, v)$ phase space, where $v$ is the velocity in the $z$ direction. The distribution is normalized by

$$
\begin{aligned}
& \int_{-\infty}^{\infty} d z \int_{-\infty}^{\infty} d v \rho(z, v, t) \\
& =\Sigma=\text { surface mass density of galaxy. }
\end{aligned}
$$

Equations of motion are given by

$$
\begin{aligned}
& \dot{z}=v \\
& \dot{v}=2 \pi G \int_{-\infty}^{\infty} d z^{\prime} \int_{-\infty}^{\infty} d v^{\prime} \rho\left(z^{\prime}, v^{\prime}, t\right) \operatorname{sgn}\left(z^{\prime}-z\right) .
\end{aligned}
$$

The Vlasov equation then reads

$$
\begin{aligned}
& \frac{\partial \rho(z, v, t)}{\partial t}+v \frac{\partial \rho(z, v, t)}{\partial z}+2 \pi G \frac{\partial \rho(z, v, t)}{\partial v} \\
& \quad \times\left[\int_{-\infty}^{\infty} d z^{\prime} \int_{-\infty}^{\infty} d v^{\prime} \rho\left(z^{\prime}, v^{\prime}, t\right) \operatorname{sgn}\left(z^{\prime}-z\right)\right]=0 .
\end{aligned}
$$

We will first have to find an equilibrium distribution $\rho_{0}(z, v)$ that satisfies the unperturbed equation

$$
\begin{aligned}
& v \frac{\partial \rho_{0}(z, v)}{\partial z}+2 \pi G \frac{\partial \rho_{0}(z, v)}{\partial v} \\
& \quad \times\left[\int_{-\infty}^{\infty} d z^{\prime} \int_{-\infty}^{\infty} d v^{\prime} \rho_{0}\left(z^{\prime}, v^{\prime}\right) \operatorname{sgn}\left(z^{\prime}-z\right)\right]=0 .
\end{aligned}
$$

A set of solutions for $\rho_{0}(z, v)$ can be found by identifying the unperturbed Hamiltonian of the system [11],

$$
H_{0}(z, v)=\frac{v^{2}}{2}+2 \pi G \int_{-\infty}^{\infty} d z^{\prime} \int_{-\infty}^{\infty} d v^{\prime} \rho_{0}\left(z^{\prime}, v^{\prime}\right)\left|z^{\prime}-z\right| .
$$

The unperturbed distribution then can be expressed as any function of $H_{0}(z, v)$. Any reasonable function of $H_{0}(z, v)$ can be a possible choice for $\rho_{0}(z, v)$, but one then notes that $H_{0}$ also depends on $\rho_{0}$, which means $\rho_{0}$ and $H_{0}$ must be solved simultaneously and self-consistently.

In this 1D model, we shall first assume that the unperturbed distribution is given by the following ansatz form:

$$
\rho_{0}(z, v)= \begin{cases}\frac{1}{2 \pi} \sqrt{\frac{\Sigma}{2 \pi G A}} \frac{1}{\sqrt{A^{2}-z^{2}-\left(\frac{A}{2 \pi G \Sigma}\right) v^{2}},}, & \text { if } z^{2}+\left(\frac{A}{2 \pi G \Sigma}\right) v^{2}<A^{2} \\ 0, & \text { if } z^{2}+\left(\frac{A}{2 \pi G \Sigma}\right) v^{2}>A^{2}\end{cases}
$$


With this $\rho_{0}$, it is easy to show that

$$
H_{0}(z, v)=\frac{v^{2}}{2}+\left(\frac{\pi G \Sigma}{A}\right)\left(A^{2}+z^{2}\right)
$$

and therefore $\rho_{0}$ is indeed a function of $H_{0}$. The choice of the ansatz distribution (A1) solves the self-consistency problem.

It also follows that the galaxy in the $z$ dimension has a uniform distribution,

$$
\int_{-\infty}^{\infty} d v \rho_{0}(z, v)= \begin{cases}\frac{\Sigma}{2 A} & \text { if }|z|<A \\ 0 & \text { if }|z|>A .\end{cases}
$$

The quantity $A$, which will remain a free parameter, is therefore the size of the galaxy in this model. This uniform distribution substantially simplifies the dynamics. In accelerator physics, this trick of finding a self-consistent and uniform beam distribution is called a KapchinskijVladimirskij distribution [12].

Consider one star in this 1D galaxy: its equations of motion in the background of the unperturbed galaxy are given by

$$
\dot{z}=v \quad \dot{v}=-\frac{2 \pi G \Sigma}{A} z .
$$

This constitutes a simple harmonic oscillation with angular frequency

$$
\omega_{0}=\sqrt{\frac{2 \pi G \Sigma}{A}} .
$$

What this means is that all stars, if considered as single particles, oscillate with the same frequency in the background of the equilibrium mass distribution of the galaxy. But we still have to consider collective perturbations.

In the present effort, we do not continue with the analytic approach, and resort to a numerical simulation. We first generate 500 stars according to the distribution (A1) in the phase space $(z, v)$. Evolution of these stars is then tracked for a period of time with $\omega_{0} T=2000$ in time steps of $\omega_{0} \Delta t=0.04$. It is found that there is no instability observed in this simulation. See Fig. 6. This 1D nonrotating galaxy is always stable because the temperature introduced in the initial distribution (A1) fulfills already the Landau stability criterion.

\section{APPENDIX B: A 2D ROTATING GALAXY MODEL}

Consider now a 2D galaxy that has a uniform infinitely long extension in $z$. All stars are now infinitely long strings, and they move only in the transverse plane perpendicular to the longitudinal $z$ axis. Let the galaxy have a star distribution $\rho(x, \dot{x}, y, \dot{y}, t)$ in the $4 \mathrm{D}$ transverse phase space $(x, \dot{x}, y, \dot{y})$. Let the unperturbed beam have a cylindrically symmetric, uniform distribution

$$
\int d \dot{x} \int d \dot{y} \rho_{0}(x, \dot{x}, y, \dot{y})= \begin{cases}\frac{\lambda_{m}}{\pi A^{2}}, & \text { if } x^{2}+y^{2}<A^{2} \\ 0, & \text { if } x^{2}+y^{2}>A^{2}\end{cases}
$$

where $A$ is the maximum transverse radial extent and $\lambda_{m}$ is the longitudinal linear mass density of the galaxy. Let the unperturbed velocity distribution be given by a rotation around the $\hat{z}$ axis with a uniform angular velocity $\omega_{0}$.

The gravitation force of this uniform cylindrical distribution is given by

$$
\frac{d^{2} \vec{r}}{d t^{2}}=-\frac{2 G \lambda_{m}}{A^{2}} \vec{r}
$$

The centrifugal force of this rotation is balanced by the gravitational attraction towards the center of the galaxy. For this to happen, the galaxy must rotate with

$$
\omega_{0}=\sqrt{\frac{2 G \lambda_{m}}{A^{2}}} .
$$

We have thus prepared a model of $2 \mathrm{D}$ rotating galaxy. Strictly speaking, in this model, there is not a temperature because the initial velocity of each star is determined by its rotation around the galaxy axis and there are no statistical thermal contributions. However, this rigid rotation of the galaxy still introduces a spread of velocities of the stars, and that velocity spread still introduces Landau damping strong enough to stabilize the galaxy in this model.

Figure 7 shows the result of a simulation for the case of 500 rotating stars. A uniform initial distribution is generated and the stars are tracked for $\omega_{0} T=1000$ or $1000 / 2 \pi$ rotation periods using a time step of $\omega_{0} \Delta t=0.04$. It can be seen that this $2 \mathrm{D}$ rotating galaxy is always stable.

[1] See, for example, Maxwell on Saturn's Rings, edited by Stephen G. Bush, C. W.F. Everitt, and Elizabeth Garber (MIT Press, Cambridge, MA, 1983).

[2] See, for examples, P. L. Palmer, Stability of Collisionless Stellar Systems (Kluwer Academic Publishers, The Netherlands, 1994); P. J.E. Peebles, Principles of Physical Cosmology (Princeton University Press, New Jersey, 1993), and references quoted therein.

[3] See, for example, Alexander W. Chao, Physics of Collective Beam Instabilities in High Energy Accelerators (John Wiley \& Sons, New York, 1993), and references quoted therein.

[4] A. A. Vlasov, J. Phys. USSR 9, 25 (1945); S. Chandrasekhar, Plasma Physics (University of Chicago, Chicago, IL, 1960).

[5] Equation (4) is allowed because, as we will do next, we will linearize the equation of motion with respect to $\Delta \rho$, and after doing so, an arbitrary perturbation can be decomposed as Fourier components as in (4) without losing generality. The step of linearization is critical here.

[6] L. D. Landau, J. Phys. USSR 10, 25 (1946).

[7] The reason of the well behavior around the singularity is because, by insisting that the range of integration along the 
real axis has a left-right symmetry about the singularity, the positive divergence below the singularity and the negative divergence above the singularity cancel each other.

[8] E. Keil and W. Schnell, CERN Report No. TH-RF/69-48, 1969.

[9] The Milky Way is a spiral rotating galaxy, but we still adopt its values as typical galaxy parameters.

[10] Alex Chao, in Proceedings of the Workshop on Quantum Aspects of Beam Physics, Hiroshima, Japan (SLAC Report No. SLAC-PUB-10019, 2003); Alexander W.
Chao, J. High Energy Phys. Nucl. Phys. 30, 6 (2006), supplement.

[11] Analysis again parallels that encountered in accelerator physics. See, for example, Frank J. Sacherer, Ph.D. thesis, University of California at Berkeley [UCRL-18454, 1968].

[12] I. M. Kapchinskij and V. V. Vladimirskij, in Proceedings of the International Conference on High Energy Accelerators and Instrumentation (CERN, Geneva, 1959), p. 274. 